\title{
Measurement of Methane Content in Upgraded Biogas Using Pulse-Echo Ultrasound
}

\author{
Johan E. Carlson ${ }^{1, *}$, Jesper Martinsson ${ }^{1}$, and Magnus Lundberg Nordenvaad ${ }^{2}$ \\ ${ }^{1}$ EISLAB, Dept. of Computer Science and Electrical Engineering, \\ Luleå University of Technology, SE-971 87 Luleå, Sweden, Johan.Carlson@1tu.se \\ ${ }^{2}$ Div. of Systems and Interaction, Dept. of Computer Science and Electrical Engineering, \\ Luleå University of Technology, SE-971 87 Luleå, Sweden
}

\begin{abstract}
In this paper we present an ultrasonic pulseecho technique for estimating the methane $\left(\mathrm{CH}_{4}\right)$ content in binary mixtures of $\mathrm{CH}_{4}$ and carbon dioxide $\left(\mathrm{CO}_{2}\right)$. The method is based on parametric estimation of phase velocity and frequency dependent attenuation in combination with Partial Least-Squares Regression (PLSR). The technique is verified using experiments on mixtures with a volume fraction of $\mathrm{CO}_{2}$ in the range of $0 \%-10 \%$. The experiments show that the $\mathrm{CH}_{4}$ content can be accurately estimated with high repeatability.
\end{abstract}

Keywords: Gas analysis, multivariate calibration, PLS regression

\section{A. Introduction}

Biogas manufactured from urban waste has been identified as a potential replacement of fossil fuels in the transport sector. The biogas is upgraded to a high methane content and then inserted into a natural gas grid. In order for this to work, the quality of the upgraded gas must be guaranteed, which means it should essentially contain only methane $\left(\mathrm{CH}_{4}\right)$ with a small fraction of carbon dioxide $\left(\mathrm{CO}_{2}\right)$, i.e. $<10 \%$ by volume.

For natural gas, which is by far the most commonly available energy gas, several measurement techniques are available, see for example [1], [2]. For other gas mixtures, like biogas or synthesis gas $\left(\mathrm{CO}, \mathrm{H}_{2}\right.$ and $\left.\mathrm{CO}_{2}\right)$, there is a lack of non-invasive on-line techniques. The long term goal of this project is to develop a method based on ultrasound for combined volume flow measurement and gas composition analysis. The method should be applicable to a wide variety of gas mixtures.

Previous work shows that the composition of a gas mixture significantly affects the acoustic wave propagation through the gas [3], [4]. Some work has also suggested how to extract these effects from experimental data using multivariate statistical tools, see [5], [6] and references therein.

In [7], it was identified that Partial Least-Squares Regression (PLSR) can be used to connect measured ultrasonic pulse spectra to the composition of mixtures of ethane and oxygen. The paper used a non-parametric method to estimate pulse spectra, which showed some limitations, mainly due to the high uncertainty of the spectral estimation, and the inherent sensitivity to experimental noise in this procedure.

This paper extends the results of [7] by estimating the frequency dependent attenuation and phase velocity of the gas mixtures using a parametric technique [8], and then using PLSR to estimate the gas composition.

\section{B. Theory}

When sound propagates through gases, two properties can be directly observed: speed of sound and attenuation. Both of these are frequency dependent. They depend on the details of underlying physics [3], [4], [9], [10]. Good physical models are crucial for the understanding of the problem, so that proper instrumentation can be designed. However, even if a good model of the underlying physics is available, the parameters of such a model are not necessarily identifiable from bandlimited ultrasound data. Furthermore, the end goal is not to describe the model, but to measure some implicit property (e.g. energy content or volume fractions). The choice of model should therefore depend on the objective of the study.

Using the pulse-echo setup described in Section C. we obtain two echoes that have traveled different distances through the gas mixture. Given these two echoes, we then estimate the frequency dependent attenuation and the phase velocity. These two quantities serve as input to a multivariate statistical calibration technique called Partial Least Squares Regression (PLSR) used to estimate the composition of the gas mixture.

\section{B.1. Attenuation and phase velocity}

Assuming linear acoustics, estimating the frequency dependent attenuation and phase velocity from ultrasound pulses essentially concerns estimation of the spectrum of a linear system $H(\omega)$, representing the gas mixture. Given the transfer function $H(\omega)$, we can calculate the attenuation $\alpha(\omega)$ and the phase velocity $c_{p}(\omega)$, where $\omega$ is the frequency (in $\mathrm{rad} / \mathrm{s}$ ). In the ultrasonic pulse-echo setup, the input and output signals $p_{1}(t)$ and $p_{2}(t)$ needed to identify $H(\omega)$ are defined as in Fig. 1.

The estimation procedure for determining $H(\omega)$ is described in detail in [8]. In addition to the estimate of $H(\omega)$, the identification procedure also yields an estimate of the covariance of the parameters of $H(\omega)$. This enables us to do the uncertainty analysis described in Sec. B.3.. 
From the estimate of $H(\omega)$ we calculate attenuation and phase velocity. Let each row, $\mathbf{x}_{n}^{T}$, of the matrix $\mathbf{X}$ be the attenuation and phase velocity corresponding to a given volume fraction of $\mathrm{CO}_{2}$, sampled at the frequencies $\omega=\omega_{k}$, where $\omega_{k}=2 \pi F_{s} k / K, k=0 \ldots K-1, K$ is the number of samples, and $F_{s}$ is the sampling frequency. The volume fraction of $\mathrm{CO}_{2}$ is then the corresponding row, $y_{n}$, of the response matrix $\mathbf{Y}$. That is

$$
\mathbf{X}=\left[\begin{array}{cc}
\boldsymbol{\alpha}_{1}^{T} & \mathbf{c}_{1}^{T} \\
\boldsymbol{\alpha}_{2}^{T} & \mathbf{c}_{2}^{T} \\
\vdots & \vdots \\
\boldsymbol{\alpha}_{N}^{T} & \mathbf{c}_{N}^{T}
\end{array}\right], \quad \mathbf{Y}=\left[\begin{array}{c}
y_{1} \\
y_{2} \\
\vdots \\
y_{N}
\end{array}\right]
$$

where $\boldsymbol{\alpha}_{n}=\left[\begin{array}{llll}\alpha\left(\omega_{0}\right) & \alpha\left(\omega_{1}\right) & \cdots & \alpha\left(\omega_{K-1}\right)\end{array}\right]^{T}, \mathbf{c}_{n}=$ $\left[\begin{array}{llll}c_{p}\left(\omega_{0}\right) & c_{p}\left(\omega_{1}\right) & \cdots & c_{p}\left(\omega_{K-1}\right)\end{array}\right]^{T}$. Here, the subscript $n$ denotes the $n$ :th row of $\mathbf{X}$ and $\mathbf{Y}$, corresponding to experiments with different volume fractions of $\mathrm{CH}_{4}$.

\section{B.2. PLS Regression}

The PLS method was developed by Herman Wold [11], and has been applied to many areas in experimental sciences. The details of the PLS calculations can be found in [7] and [12]. Here, only a brief summary is given.

The central idea of PLS, as opposed to principal component regression (PCR) and ordinary least-squares (OLS) estimation [13], is that PLS determines a set of basis functions (PLS components) for both the $\mathrm{X}$ block and the $\mathrm{Y}$ block, in such a way that they best describe the crosscovariance between the blocks. In other words, instead of looking at variations in phase velocity and attenuation alone (the $\mathrm{X}$ block), we look at variation in $\mathbf{X}$ that correlates with variation in $\mathbf{Y}$.

\section{B.2.1. Predicting $Y$ from $X$}

Using Eqs. (3)-(11) in [7], the X block and Y block are first transformed into their PLS component representation,

$$
\begin{aligned}
& \mathbf{X}=\mathbf{T} \mathbf{P}^{T}+\mathbf{E} \\
& \mathbf{Y}=\mathbf{T} \mathbf{Q}^{T}+\mathbf{F},
\end{aligned}
$$

where $\mathbf{E}$ and $\mathbf{F}$ are residual matrices of the $\mathrm{X}$ and $\mathrm{Y}$ blocks, respectively. Given an existing set of PLS components, the estimate of $\mathbf{Y}$ is given by

$$
\widehat{\mathbf{Y}}=\mathbf{X} \tilde{\mathbf{W}} \mathbf{Q}^{T},
$$

where $\tilde{\mathbf{W}}$ and $\mathbf{Q}$ are given by Eqs. (15) and (11) in [7], respectively. Here, the matrix $\tilde{\mathbf{W}} \mathbf{Q}^{T}$ is determined from a calibration experiment, and later used to estimate $\widehat{\mathbf{Y}}$.

\section{B.2.2. Determining PLS model order}

As in all modeling, finding an appropriate model order is an important, and difficult, problem. There are numerous criteria available for model order selection, some which tend to overestimate the model-order, while others are overly conservative [14]. For PLS modeling problems, cross-validation is the most commonly used method [15].

In this paper we have one training data set to estimate a PLS model and one validation set. We evaluate the model by looking at the $Q^{2}$ statistic [16] as a function of the number of components in the PLS model. For our experiments, we found that two PLS components is the best model order, which yields a $Q^{2}$ value of 0.9998 .

\section{B.3. Uncertainty analysis}

The phase velocity and attenuation for each gas composition are estimated using the parametric model in [8]. Along with these estimates we also obtain a covariance matrix for the parameters of $H(\omega)$. This enables us to generate a randomized distribution of the attenuation and phase velocity curves, and eventually also a randomized distribution of the estimated gas compositions.

Given the covariance matrix for the model parameters, the following procedure is applied in order to obtain a randomized distribution of the estimated gas compositions:

1. Randomize a large number of model parameter vectors, given the covariance matrix, $\mathbf{C}_{\boldsymbol{\theta}}$, where $\boldsymbol{\theta}$ is a vector with the parameters of the model $H(\omega)$ (i.e. the prior distribution).

2. Calculate the attenuation and phase velocity corresponding to each of the models.

3. For each randomized model, run the PLSR as described above, to obtain an empirical (posterior) distribution of the estimated gas compositions.

4. Estimate the uncertainty from the empirical distributions obtained in step 4.

Note that this procedure incorporates both uncertainty due to the noise in the ultrasonic measurements, as well as estimation errors from the PLSR step.

\section{Experiments}

\section{C.1. Experimental design}

In order to evaluate the performance of the proposed method, a series of experiments was designed. For all measurements, we used calibration gas mixtures from Air Liquide Gas AB (Kungsängen, Sweden) with an analysis uncertainty of $1 \%$ (relative to the $\mathrm{CO}_{2}$ content).

Experiments were made at a static pressure of 8 bar at room temperature (see details in the next section), for volume fractions of $\mathrm{CO}_{2}$ of $0 \%, 2 \%, 4 \%, 6 \%, 8 \%$, and $10 \%$, respectively. The order of the experiments was randomized in order to avoid misinterpretations due to systematic variations in any ambient variables beyond our control.

\section{C.2. Setup}

For all the experiments in this paper, the ultrasound echoes were measured in a pulse-echo configuration (see Fig. 1). A $300 \mathrm{kHz}$ air transducer (D-Flow Technology $\mathrm{AB}$, Luleå, Sweden) was mounted in a measurement cell, transmitting pulses through the gas towards a spherical stainless steel reflector. As input to the phase velocity and attenuation estimation algorithm, the second and fourth echo, as indicated in Fig. 1, were used. The reason for not using the first echo was that it contained some traces from the excitation. The fourth echo was exploited instead 

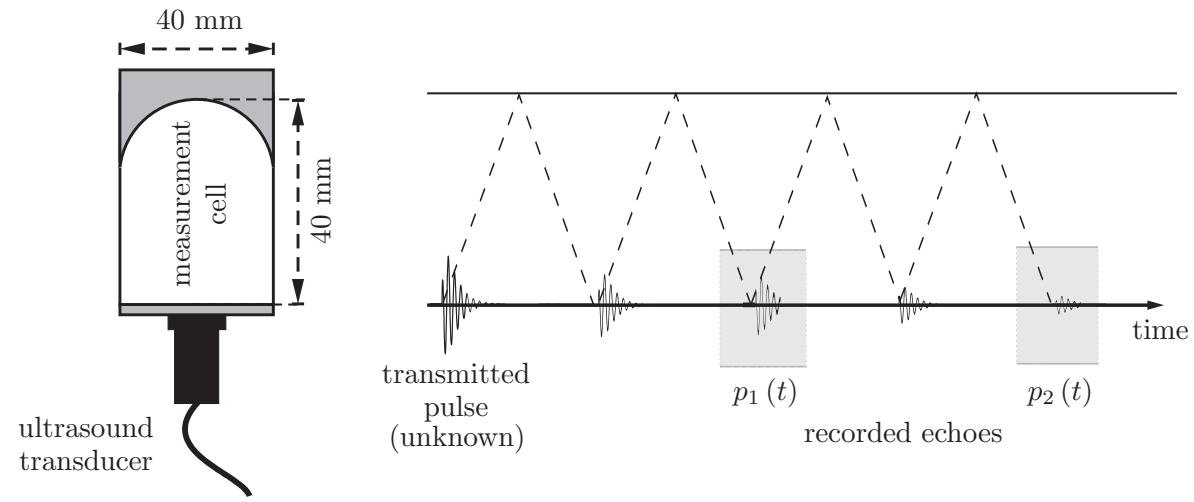

Fig. 1. The measurement cell and the pulse-echo principle. The transducer emits an unknown sound wave. The reflections from the bottom of the measurement cell is then recorded. Consecutive echoes can be recorded, as indicated in the figure. In this paper, the second and the fourth echo are used, denoted $p_{1}(t)$ and $p_{2}(t)$, respectively.

of the third in order to maximize the propagation path and thus amplify the effects of dispersion and attenuation in the gas mixture.

A custom-built pressure chamber was used to achieve the desired static pressures. For the experiments presented here, the static pressure was set to $8.0 \mathrm{bar} \pm 0.01$ bar. The pressure in the chamber was measured with an ANDERSON TPP Pressure Transmitter.

To excite and receive ultrasound pulses, a Panametrics Pulser/Receiver Model 5052 was used. In transmitting mode, the pulser/receiver was set to deliver maximum energy to the transducer, which corresponds to a short voltage peak with $380 \mathrm{~V}$ amplitude with an energy of $104 \mu \mathrm{J}$. In the measurements presented here, the gain of the pulser's receiver input was set to $40 \mathrm{~dB}$.

All pulses were sampled at $100 \mathrm{MHz}$ using a 14bit CompuScope 14100 oscilloscope card (Gage Applied Technologies Inc., Lachine, QC Canada). The pulses where later down-sampled 12 times off-line to reduce the amount of data to process.

For each measurement, the temperature was recorded using an encapsulated PT100 probe mounted through the wall of the pressure chamber. The average temperature of the gas mixtures throughout the measurements was $20.4{ }^{\circ} \mathrm{C}$ $\pm 0.2{ }^{\circ} \mathrm{C}( \pm \sigma)$, where $\sigma$ is the standard deviation of all measured temperatures.

\section{Results}

Figures 2 and 3 show the results of from the parametric estimation of attenuation and phase velocity, respectively. Fig. 2 shows the result for pure $\mathrm{CH}_{4}$ and for a mixture containing $10 \% \mathrm{CO}_{2}$ and $90 \% \mathrm{CH}_{4}$. Fig. 2 also presents the corresponding $\pm 2 \sigma$ intervals, estimated using the procedure described in Sec. B.3.. For the phase velocity, Fig. 3 illustrates all estimates. From the figure we see that there is a significant change in phase velocity as the composition of the gas mixture changes. In Fig. 3 no uncertainty intervals are shown, for the simple reason that they are much too narrow to be visible in the plot.

The attenuation and phase velocity estimates then served as input to the PLSR step. The PLS model was built from one experiment series, and the estimates in Fig. 4 were ob-

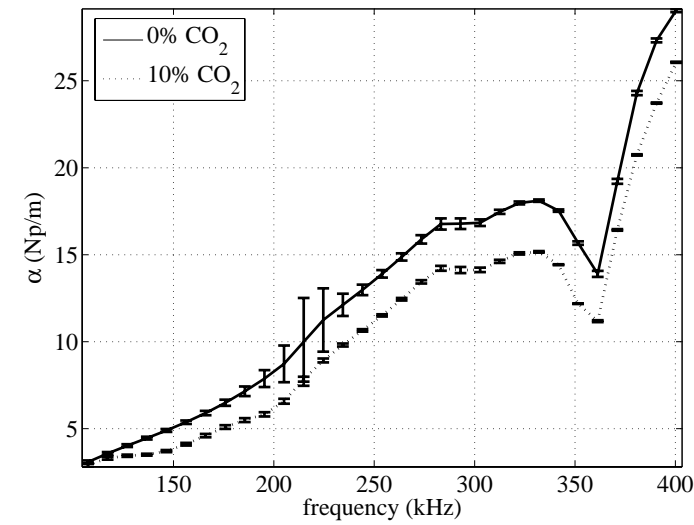

Fig. 2. Estimated attenuation coefficients $\alpha$ (in $\mathrm{Np} / \mathrm{m}$ ) as a function of the frequencies present in the pulse, for $0 \%$ and $10 \%$ $\mathrm{CO}_{2}$. The error bars indicate the $\pm 2 \sigma$ uncertainties. The corresponding curves for volume fractions of $2 \%, 4 \%, 6 \%$, and $8 \%$ lie in between these curves and have been left out for the sake of clarity.

tained using a repeated experiment. Fig. 4 shows that accurate estimates of the $\mathrm{CO}_{2}$ volume fractions were obtained. Studying the uncertainty intervals $( \pm 2 \sigma)$ we also note that the resolution is excellent, enabling the measurement of small changes in $\mathrm{CO}_{2}$ content.

\section{E. Discussion}

The use of a spherical reflector in the measurement cell (see Fig. 1) could cause bias errors in the estimates of phase velocity and attenuation, since the distance from the transducer surface to the reflector is not unambiguously known in the current setup. For this reason, we can not claim that the measurements of phase velocity and attenuations are correct, to an absolute value. However, since the PLS regression step explores variations in these properties and not absolute values, the nature of the setup should not affect the final estimate of the $\mathrm{CO}_{2}$ and $\mathrm{CH}_{4}$ content.

As in all ultrasound measurement systems, environmental factors such as pressure and temperature fluctuations, will affect the measured ultrasound pulses. Pressure and temperature can be measured and incorporated into the 


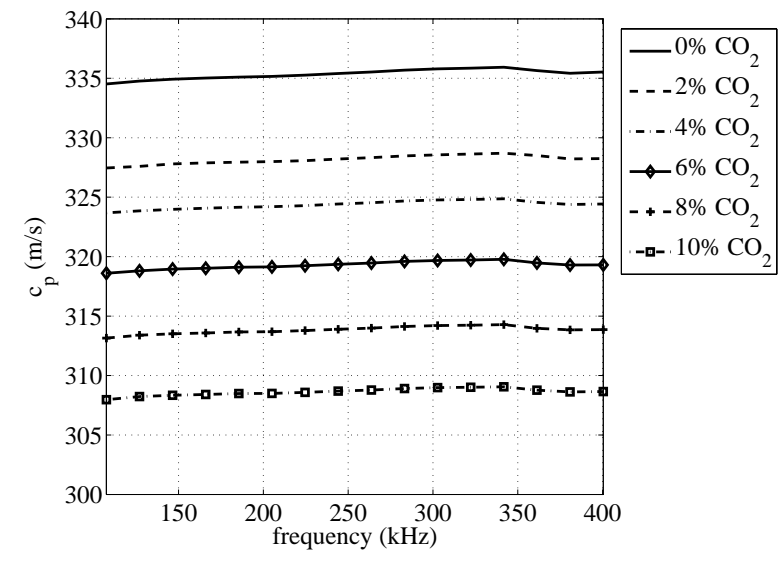

Fig. 3. Estimated phase velocities $c_{p}$ (in $\left.\mathrm{m} / \mathrm{s}\right)$. The figure shows the average value over 10000 estimates. The uncertainties are too small to be shown in the plot.

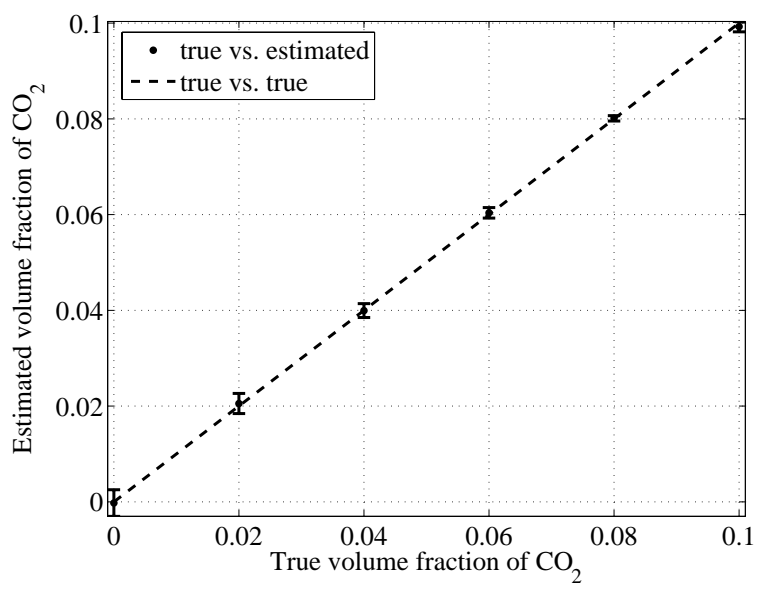

Fig. 4. True vs. estimated volume fractions of $\mathrm{CO}_{2}$. The error bars indicate the $\pm 2 \sigma$ interval for each estimated volume fraction.

PLS regression model, or if their effects are known, into the estimates of the attenuation and phase velocity. Even if changes in pressure and temperature are monitored, they are likely to decrease the performance of the estimator unless they are properly taken care of. This will be addressed in future research. However, for the small changes observed in the experiments presented in this paper, no significant effect on the estimated volume fractions should be expected.

\section{F. Conclusions}

In this paper we have demonstrated how the principle of PLS regression (PLSR) can be combined with parametric estimation of frequency dependent attenuation and phase velocity to obtain accurate estimates of the quality of upgraded biogas. We show that under constant pressure and temperature conditions, the volume fraction of $\mathrm{CO}_{2}$ can be accurately estimated with high repeatability, using a technique based solely on ultrasonic pulse-echo measurements. Since the gas is a binary mixture of $\mathrm{CO}_{2}$ and $\mathrm{CH}_{4}$, this also gives an estimate of the $\mathrm{CH}_{4}$ content, which is the dominant factor when assessing the quality of upgraded biogas.

\section{G. Acknowledgements}

The research was performed under grants from the Swedish Energy Agency and the Kempe Foundation.

\section{H. Literature}

[1] M. Jaeschke, P. Schley, and R. J. van Rossmalen, "Thermodynamic research improves energy measurement in natural gas,” Int. J. Thermophys., vol. 23, no. 4, pp. 1013-1031, 2002.

[2] M. Jaeschke, "Thermodynamic research improves energy measurement of natural gas," Thermochimica Acta, vol. 382, pp. 37-45, 2002.

[3] Y. Dain and R. M. Lueptow, "Acoustic Attenuation in Three-Component Gas Mixtures - Theory," J. Acoust. Soc. Am., vol. 109, pp. 1955-1964, 2001.

[4] Y. Dain and R. M. Lueptow, "Acoustic Attenuation in Three-Component Gas Mixtures - Results," J. Acoust. Soc. Am., vol. 110, pp. 2974-2979, 2001.

[5] J. E. Carlson and P.-E. Martinsson, "Exploring interaction effects in two-component gas mixtures using orthogonal signal correction of ultrasound pulses," J. Acoust. Soc. Am., vol. 117, no. 5, pp. 2961-2968, 2005.

[6] P.-E. Martinsson and J. E. Carlson, "Investigating the Feasibility of Using Principal Component Analysis for Ultrasonic Classification of Gas Mixtures," in Proc. IEEE Int. Ultrason. Symp., (Honolulu, Hawaii, USA), pp. 1396-1399, IEEE, Oct. 5-8 2003.

[7] J. E. Carlson and R. Carlson, "Prediction of molar fractions in two-component gas mixtures using pulse-echo ultrasound and PLS regression," IEEE Trans. Ultrason., Ferroelec., and Freq. Contr., vol. 53, no. 3, pp. 606-613, 2006.

[8] J. Martinsson, J. E. Carlson, and J. Niemi, "Model-based phase velocity and attenuation estimation in wideband ultrasonic measurement systems," IEEE Trans. Ultrason., Ferroelec., and Freq. Contr., vol. 54, pp. 138-146, Jan. 2007.

[9] P.-E. Martinsson and J. Delsing, "Ultrasonic Measurements of Molecular Relaxation in Ethane and Carbon Monoxide," in Proc. IEEE Int. Ultrason. Symp., (Munich, Germany), pp. 494-499, IEEE, October 8-11 2002.

[10] A. B. Bhatia, Ultrasonic Absorption - An Introduction to the Theory of Sound Absorption and Dispersion in Gases, Liquids and Solids. New York: Dover Publications, 1985.

[11] H. Wold, Systems Under Indirect Observation, Vols I, II. Amsterdam: North Holland, 1982.

[12] S. Wold, M. Sjöström, and L. Eriksson, "PLS regression: A basic tool of chemometrics," Chemometrics Intell. Lab. Syst., vol. 58, pp. 109-130, 2001.

[13] I. T. Jolliffe, Principal Component Analysis. New York: Springer Verlag, 2nd ed., 2002.

[14] J. Shao, "Linear model order selection by cross-validation," J. Am. Stat. Assoc., vol. 88, pp. 486-494, June 1993.

[15] I. N. Wakeling and J. J. Morris, "A test of significance for partial least squares regression," J. Chemom., no. 7, pp. 291-304, 1993.

[16] R. Carlson and J. E. Carlson, Design and Optimization in Organic Synthesis: Second Enlarged and Revised Edition. Amsterdam: Elsevier, 2nd ed., 2005. 